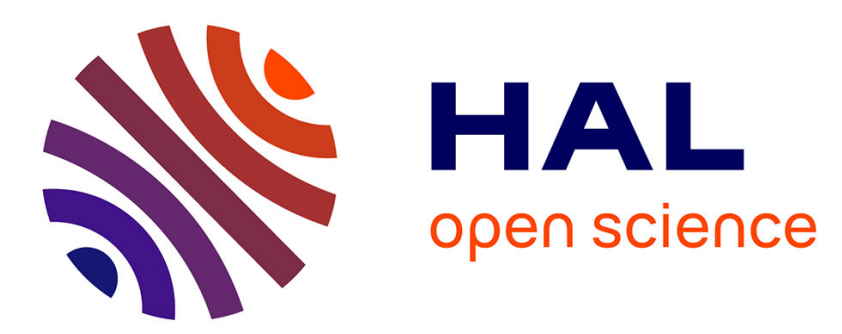

\title{
High speed pulsed electrical spin injection in spin-light emitting diode
}

\author{
V. G Truong, P.-H Binh, Pierre Renucci, M. Tran, Y. Lu, H. Jaffres, J.-M. \\ George, C. Deranlot, A. Lemaitre, Thierry Amand, et al.
}

\section{- To cite this version:}

V. G Truong, P.-H Binh, Pierre Renucci, M. Tran, Y. Lu, et al.. High speed pulsed electrical spin injection in spin-light emitting diode. Applied Physics Letters, 2009, 94 (141109), 10.1063/1.3110990 . hal-01759258

\section{HAL Id: hal-01759258 \\ https://hal.science/hal-01759258}

Submitted on 5 Apr 2018

HAL is a multi-disciplinary open access archive for the deposit and dissemination of scientific research documents, whether they are published or not. The documents may come from teaching and research institutions in France or abroad, or from public or private research centers.
L'archive ouverte pluridisciplinaire HAL, est destinée au dépôt et à la diffusion de documents scientifiques de niveau recherche, publiés ou non, émanant des établissements d'enseignement et de recherche français ou étrangers, des laboratoires publics ou privés. 


\title{
High speed pulsed electrical spin injection in spin-light emitting diode
}

\author{
V. G. Truong, ${ }^{1}$ P.-H. Binh, ${ }^{2}$ P. Renucci, ${ }^{1, a)}$ M. Tran, ${ }^{3}$ Y. Lu, ${ }^{3}$ H. Jaffrès, ${ }^{3}$ J.-M. George,${ }^{3}$ \\ C. Deranlot, ${ }^{3}$ A. Lemaître, ${ }^{4}$ T. Amand ${ }^{1}$ and X. Marie ${ }^{1}$ \\ ${ }^{1}$ Université de Toulouse, INSA-CNRS-UPS, LPCNO, 135 Avenue de Rangueil, 31077 Toulouse, France \\ ${ }^{2}$ Institute of Material Science, 18 Hoang Quoc Viet Road, Cau Giay Dist, Hanoï 10000, Vietnam \\ ${ }^{3}$ Unité Mixte de Physique CNRS/Thales, RD 128, 91767 Palaiseau, France and Université Paris-Sud 11, \\ 91405 Orsay, France \\ ${ }^{4}$ CNRS-Laboratoire de Photonique et Nanostructures, Route de Nozay, 91460 Marcoussis, France
}

(Received 22 December 2008; accepted 10 March 2009; published online 10 April 2009)

\begin{abstract}
We demonstrate high speed pulsed electrical spin injection from a $\mathrm{CoFeB} / \mathrm{MgO}$ spin injector into a $\mathrm{AlGaAs} / \mathrm{GaAs}$ semiconductor light emitting diode. Under pulsed electrical excitation, time-resolved electroluminescence on nanosecond time scale exhibits a plateau of circular polarization degree as high as $15 \%$ under a $0.8 \mathrm{~T}$ magnetic field. It follows an initial decay that could be due to electron spin-relaxation process in the quantum well embedded in the intrinsic region of the diode. The temporal buildup of the electronic spin polarization degree in the quantum well is much faster than the rise time of electroluminescence intensity. (C) 2009 American Institute of Physics.
\end{abstract}

[DOI: $10.1063 / 1.3110990]$

The manipulation of the spin degree of freedom in semiconductors could yield to devices with additional functionalities. Various experiments have been performed in order to electrically generate ${ }^{1}$ and control spin-polarized carriers in semiconductors, in particular for high speed applications. ${ }^{2}$ Besides these schemes, a promising one could consist in injecting spin-polarized currents in pulsed regime from a ferromagnetic (FM) source into a semiconducting heterostructure. For this purpose, an efficient injection of spin-polarized electrons can be obtained by the insertion of a thin tunnel barrier $^{3}$ at the interface between both types of materials. In particular $\mathrm{MgO}$ tunnel barriers yield very efficient spin injection, ${ }^{4-6}$ thanks to the symmetry selection rules which govern the tunneling transfer of carriers. ${ }^{7}$ One of the most powerful tools to estimate the spin injection efficiency is based on the optical detection ${ }^{8-10}$ in devices called spin-light emitting diodes (spin-LEDs) with a quantum well (QW) embedded in the intrinsic region of the diode. The electron spin polarization within the semiconductor can be inferred from the measured circular polarization of the electroluminescence signal (EL) through optical selection rules ${ }^{11}$ for a QW. To the best of our knowledge, only quasistationary $(\mathrm{cw})$ operating mode for electrical spin injection has been evidenced up to now by EL measurements. For future applications, it is also important to demonstrate very high speed pulsed operation. This could appear as a cornerstone for the generation of high speed spin currents as well as the fast initialization of quantum memories based on spin.

In this letter, we explore the ability of such device to be operated under pulsed electrical excitation by time-resolved EL, and we report on the ultrafast electrical injection of spinpolarized electrons in a $\mathrm{CoFeB} / \mathrm{MgO} / \mathrm{AlGaAs}$ structure. A quasiconstant circular polarization degree $\left(P_{c}\right)$ as large as $15 \%$ at $T=15 \mathrm{~K}$ for a magnetic field of $0.8 \mathrm{~T}$ under electrical pulsed excitation is evidenced during the very short nanosecond window of light emission. This plateau behavior for $P_{c}$ occurs after an initial decay that could be due to electron

${ }^{a)}$ Electronic mail: pierre.renucci@insa-toulouse.fr. spin-relaxation process. In addition, it is established that the electron spin polarization reaches its maximum value before the first 700 ps of the EL emission.

The spin-polarized light emitting diode structure [see Fig. 1(a)] was grown by molecular beam epitaxy (MBE) for the semiconductor part before depositing the tunnel barrier/ferromagnet contact by sputtering method. The $p-i-n$ LED device has the following structure sequence: $p$-GaAs:Zn (001) substrate $\left(p=2 \times 10^{19} \mathrm{~cm}^{-3}\right) / 500 \mathrm{~nm}$ $p-\mathrm{Al}_{0.08} \mathrm{Ga}_{0.92} \mathrm{As}: \mathrm{Be}\left(p=1.7 \times 10^{19} \mathrm{~cm}^{-3}\right) / 50 \mathrm{~nm}$ undoped $\mathrm{Al}_{0.08} \mathrm{Ga}_{0.92} \mathrm{As} / 10 \mathrm{~nm}$ undoped $\mathrm{GaAs} / 50 \mathrm{~nm}$ undoped $\mathrm{Al}_{0.08} \mathrm{Ga}_{0.92} \mathrm{As} / 50 \mathrm{~nm} \quad n-\mathrm{Al}_{0.08} \mathrm{Ga}_{0.92} \mathrm{As}: \mathrm{Si} \quad(n=1$ $\times 10^{16} \mathrm{~cm}^{-3}$ ). The LED was passivated with arsenic in the MBE chamber and then transferred in air into a magnetron sputtering system to grow the $\mathrm{CoFeB} / \mathrm{MgO}$ spin injector. $\mathrm{Be}$ fore that, the As capping layer was first desorbed at $400{ }^{\circ} \mathrm{C}$ by in situ reflection high energy electron diffraction control in a separate chamber connected to the main sputtering chamber. The spin injector consists of a $\mathrm{MgO}$ tunnel barrier

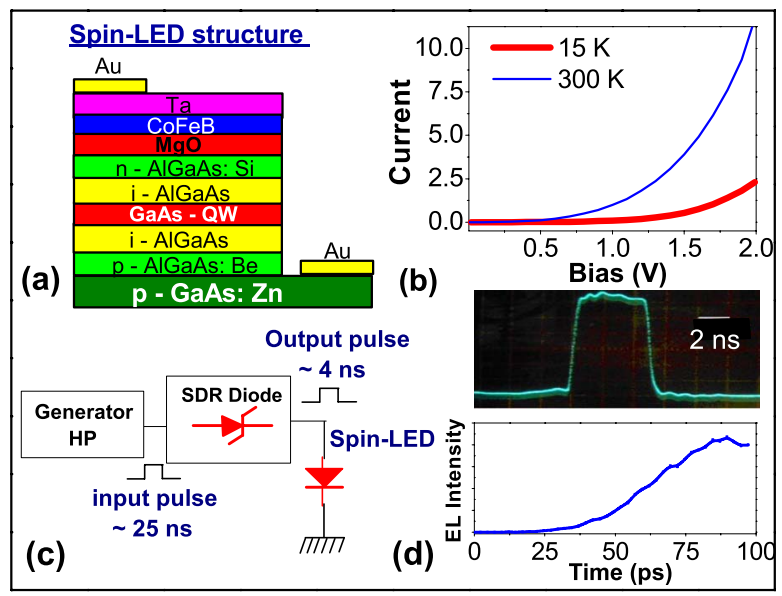

FIG. 1. (Color online) (a) Spin-LED structure. (b) $I(V)$ characteristics at 15 $\mathrm{K}$ (thick line) and $300 \mathrm{~K}$ (thin line). (c) Scheme of the high speed electrical pulse generator. (d) Up: time evolution of electrical pulse measured by a fast sampling oscilloscope in the 2 ns range. Down: rising edge of the EL of a VCSEL measured by the streakscope with a temporal resolution of $25 \mathrm{ps}$. 
with a thicknesses of $2.6 \mathrm{~nm}$ grown at $200{ }^{\circ} \mathrm{C}$, followed by a $3 \mathrm{~nm} \mathrm{Co}{ }_{40} \mathrm{Fe}_{40} \mathrm{~B}_{20} \mathrm{FM}$ contact capped with $5 \mathrm{~nm}$ Ta layer to prevent oxidation. The sputtering conditions for $\mathrm{MgO}$ and metals can be found elsewhere. ${ }^{12}$ Circular mesas with $300 \mu \mathrm{m}$ diameter were then processed using standard photolithography and etching techniques.

The EL measurements are performed at low temperature $(15 \mathrm{~K})$ in a helium closed-cycle cryostat. For quasi-cw EL, the sample is excited with square pulses of $5 \mu$ s at $50 \mathrm{kHz}$. For time-resolved EL, an electrical impulse generating circuit using a step recovery diode (SRD) (Ref. 13) from MMD-0840 Aeroflex-Metelics Corporation, is inserted in the cryostat and driven by a square-pulsed generator with a pulse duration of $25 \mathrm{~ns}$ and rise and fall times of $3 \mathrm{~ns}$ [Fig. 1(c)]. The repetition rate is $2 \mathrm{MHz}$. We have characterized the impulse forming circuit with a fast sampling oscilloscope (Tektronic 3S1 sampling head) with a temporal resolution of 350 ps [Fig. 1(d)]: the square pulse duration is $4 \mathrm{~ns}$. For the polarization resolved EL measurements, the spin-LED is placed into a Helmoltz-split magnetic coil providing a maximum magnetic field $(B)$ of $0.8 \mathrm{~T}$ normal to the sample plane. The EL signal is detected in the Faraday geometry. The EL circular polarization degree $P_{c}$ is analyzed through a $\lambda / 4$ wave plate and a linear analyzer and is defined as $P_{C}=\left(I^{\sigma+}\right.$ $\left.-I^{\sigma-}\right) /\left(I^{\sigma+}+I^{\sigma-}\right)$ where $I^{\sigma+}$ and $I^{\sigma-}$ are the intensities of the right $\left(\sigma^{+}\right)$and left $\left(\sigma^{-}\right)$circularly polarized components of the luminescence, respectively. Two different ways of detection are available: (i) a very low noise charged-coupled device with a monochromator is used for $\mathrm{cw}$ measurements (the spectral resolution of the whole setup is of about 2 $\mathrm{meV}$ ); (ii) a streakscope with a S20 photocathode is synchronized with the pulsed generator to measure the time resolved EL with a temporal resolution of 125 ps (for the range used for the measurements in this paper) in photon counting mode. Using the latter detector, we have characterized the electrical pulse provided by the impulse forming circuit through the EL of an ultrafast vertical cavity surface emitting laser (VCSEL) $850 \mathrm{~nm}$ (HFE4080-321-XBA, Honeywell). The rise [see Fig. 1(d)] and fall times are estimated about $100 \pm 25$ ps (the streakscope is used with its fastest time range, corresponding to a temporal resolution of $25 \mathrm{ps}$ ), that is consistent with the transition time of 75 ps of the SRD embedded in the impulse forming circuit.

Figure 2 shows typical cw EL spectra acquired at $15 \mathrm{~K}$ under $V=2.9 \mathrm{~V}$ bias for $B=0$ [inset (b) in Fig. 2] and $0.8 \mathrm{~T}$ [Fig. 2(a)]. Whereas the heavy-hole exciton (XH) EL peak observed at $812 \mathrm{~nm}$ does not show any circular polarization at zero magnetic field, the EL polarization reaches $21 \% \pm 2 \%$ under $0.8 \mathrm{~T} .{ }^{14}$ According to the optical selection rules applied to $\mathrm{QW},{ }^{11}$ the EL circular polarization $P_{C}$ is proportional to the electron spin polarization $P_{E}\left(P_{C}=F P_{E}\right)$. The factor $F$ $=1 /\left(1+\tau / \tau_{s}\right)$ takes into account the electron spin relaxation $\tau_{s}$ during its lifetime $\tau$ in the QW. ${ }^{6,15-17} F$ is estimated to be around $0.50 \pm 0.03$ by independent measurements of $\tau_{s}$ $(\approx 450 \pm 35$ ps at $1 / e)$ and $\tau(\approx 450 \pm 20$ ps at $1 / e)$ by timeresolved photoluminescence ${ }^{6}$ (TRPL). The time evolution of the photoluminescence degree of circular polarization $P_{c}$ (after a $1.5 \mathrm{ps}\left(\sigma^{+}\right)$laser pulsed excitation at $\left.1.653 \mathrm{eV}\right)$ is displayed in the inset in Fig. 3(b). From this measurement, it is possible to extract $\tau_{s} \approx 450 \pm 35 \mathrm{ps}$. So the electrons spin polarization $P_{E}$ in the semiconductor part, before electrons are trapped in the $\mathrm{QW}$ can be estimated to be about $P_{E}$

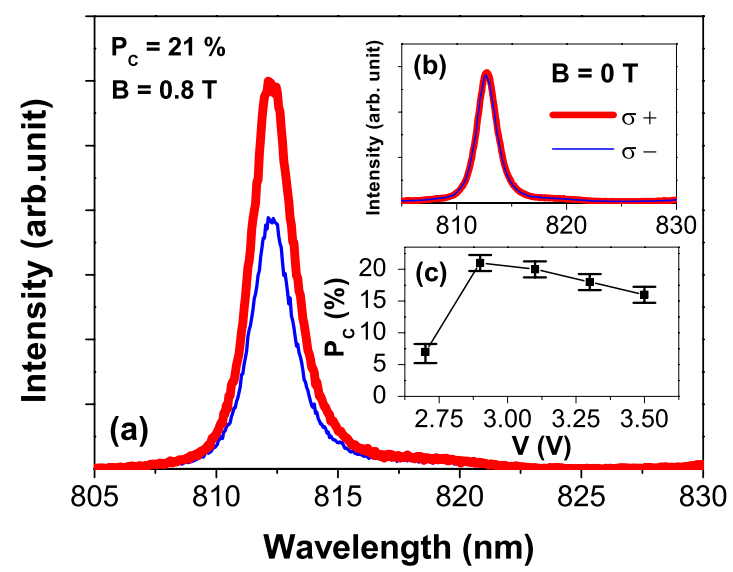

FIG. 2. (Color online) (a) EL spectra at $15 \mathrm{~K}$ under an external magnetic field of $0.8 \mathrm{~T}$ for $I^{\sigma+}$ (thick line) and $I^{\sigma-}$ (thin line) EL components. Inset (b) same quantities for $B=0 \mathrm{~T}$. Inset (c) bias dependence of the EL polarization degree $P_{C}(B=0.8 \mathrm{~T})$.

$=42 \% \pm 6 \%$. Let us note that the saturation field for CoFeB measured by superconducting quantum interference device (SQUID) magnetometer measurements is $1.3 \mathrm{~T}$. An extrapolation procedure by matching the EL polarization results with the SQUID data in the linear regime ${ }^{6}$ allows a rough estimate of $P_{E}$ at saturation; we find $P_{E} \sim 68 \% \pm 10 \%$, proving the very high efficiency of $\mathrm{CoFeB} / \mathrm{MgO}$ spin injector. The bias dependence of $P_{C}$ is shown in the inset (c) of Fig. 2. After a first increase toward a maximum regime of $21 \%, P_{C}$ monotonously decreases with the applied voltage. This trend has also been observed by Adelmann et al. ${ }^{17,18}$ and Dong et al. ${ }^{19}$ for different FM spin injectors. These authors attribute this dependence to the variation in the ratio $\tau / \tau_{s}$ as a function of the applied voltage.

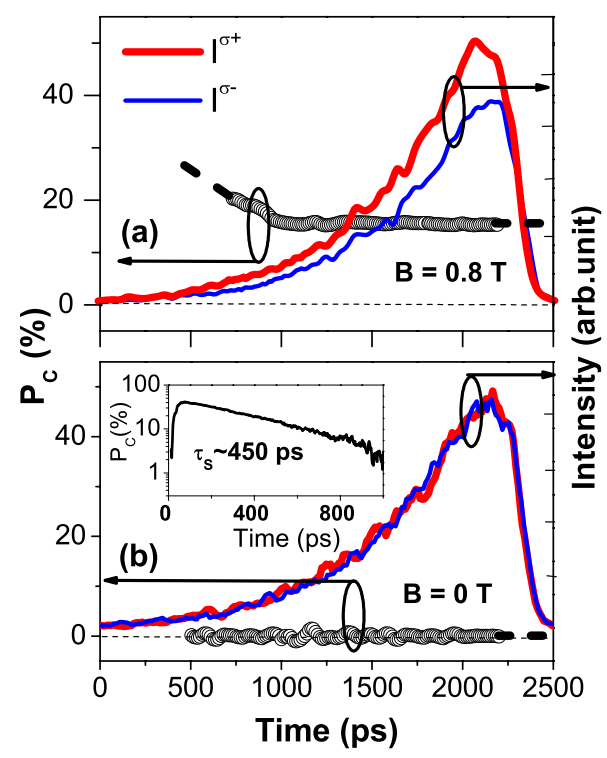

FIG. 3. (Color online) (a) Right axis: time-resolved EL intensity at $T$ $=15 \mathrm{~K}$ of the $\sigma+$ (thick line) and $\sigma^{-}$(thin line) polarized components with an applied bias $V=4 \mathrm{~V}$ for $B=0.8 \mathrm{~T}$. Left axis: time evolution of the EL circular polarization degree $P_{C}$ (open circles). The dotted line is a guide for the eyes. (b) Right axis: time-resolved EL intensity at $T=15 \mathrm{~K}$ of the $\sigma+$ (thick line) and $\sigma^{-}$(thin line) polarized components with an applied bias $V=4 \mathrm{~V}$ for $B=0 \mathrm{~T}$. Left axis: time evolution of the EL circular polarization $P_{C}$ (open circles). Inset: time evolution of the circular polarization degree $P_{C}$ of the PL at $T=15 \mathrm{~K}$ after a $1.5 \mathrm{ps}(\sigma+)$ laser pulsed excitation at 1.653 eV (above AlGaAs gap). 
After the characterization of spin injection by $\mathrm{cw}$ and spectrally resolved EL, we turn now to the study of timeresolved EL in the nanosecond range performed on the XH peak. Figure 3 (right axis) shows time-resolved EL spectra for $I^{\sigma+}$ and $I^{\sigma-}$ intensities acquired at $15 \mathrm{~K}$ for $B=0.8 \mathrm{~T}$ (the applied bias is $V=4 \mathrm{~V}$ ). The time origin $t=0$ is chosen when the EL signal appears, that is $1700 \mathrm{ps}$ after the rising edge of the electrical pulse. We observe first an increase of the EL signal with a rise time of about $1250 \pm 125$ ps (measured between $10 \%$ and $90 \%$ of maximum signal), followed by a sudden drop with a characteristic time at $(1 / e)$ of $200 \pm 125 \mathrm{ps}$, close to the temporal resolution of our setup. This slow EL rise time, compared to the fast rise time (100 ps) of the applied voltage, is due to parasitic resistances, inductances, and capacitances of the device. The temporal window for EL emission (around $2.4 \mathrm{~ns}$ at the foot of the EL pulse) is shorter than the electrical pulse duration (about 4 ns): it could be due to the fact that the threshold voltage for EL emission is only reached for this window. The very fast quenching of the EL signal in 200 ps indicates that the lifetime of carriers during the fall of the electrical pulse is no more governed by radiative recombinations. This is opposite to the case of TRPL measurements under pulsed laser excitation where a characteristic lifetime at $(1 / e) \tau$ of $450 \pm 35$ ps was previously measured ${ }^{6}$ at $15 \mathrm{~K}$. The fast decrease of EL intensity could be ascribed to the sweep of carriers out of the QW corresponding to a short reverse peak current at the end of the electrical pulse. The analysis of this dynamical behavior is currently under investigation and is beyond the scope of this paper. The time evolution of the EL circular polarization degree $P_{c}$ can be deduced from $I^{\sigma+}$ and $I^{\sigma-}$ intensities and is displayed on the left axis of Fig. 3. When a longitudinal magnetic field of $0.8 \mathrm{~T}$ is applied, an EL circular polarization is evidenced [Fig. 3(a)], whereas no circular polarization can be detected for $B=0$ [Fig. 3(b)]. This demonstrates a rapid establishment of the current-spin polarization below the nanosecond scale. Due to a poor signal to noise ratio, it is however not possible to determine $P_{C}(t)$ during the first 700 ps of the dynamics. From 700 ps to 1000 ps, $P_{c}$ decreases and then remains quasiconstant $(\approx 15 \% \pm 3 \%)$ during the whole dynamics. This could be due to electron spin relaxation in the QW measured previously, and could be responsible for the circular polarization decrease during the rising time of EL, before the system reaches a quasistationary regime after around $1 \mathrm{~ns}$ (from the point of view of $P_{c}$ evolution). The presence of a large circular polarization as soon as any EL signal is detectable proves unambiguously that the build-up time of the electronic spin polarization in the QW embedded in the intrinsic region of the diode is much faster than the rise time of EL signal. The former takes place on a time scale at least shorter than $700 \mathrm{ps}$.

In conclusion, we have demonstrated high speed pulsed electrical spin injection from $\mathrm{CoFeB} / \mathrm{MgO}$ spin injector into a $\mathrm{AlGaAs} / \mathrm{GaAs}$ semiconductor light emitting diode. This injection is characterized by an EL circular polarization plateau as high as $15 \%$ during the short nanosecond windows of light emission at $15 \mathrm{~K}$ under a $0.8 \mathrm{~T}$ magnetic field. This plateau occurs after an initial decay that may be due to electron spin-relaxation process in the QW embedded in the intrinsic region of the diode. We observe that the rise time of electronic spin polarization rate in the $\mathrm{QW}$ is much faster than the rise time of EL. Note that the time response of the device could be shortened by reducing the size of the mesa and by using an undoped substrate with air bridges to reduce its capacitance. This could allow the excitation of the device with electrical pulses shorter than $250 \mathrm{ps}$ in order to follow more accurately the spin dynamics as well as to get the electrical spin injection at a higher repetition rate.

We thank A. Balocchi and B. Urbaszek for fruitful discussions. This work is partially supported by the French Agence Nationale pour la Recherche (ANR), contract MOMES.

${ }^{1}$ V. Sih, W. H. Lau, R. C. Myers, V. R. Horowitz, A. C. Gossard, and D. D. Awschalom, Phys. Rev. Lett. 97, 096605 (2006).

${ }^{2}$ N. P. Stern, D. W. Steuerman, S. Mack, A. C. Gossard, and D. D. Awschalom, Nat. Phys. 4, 843 (2008).

${ }^{3}$ A. Fert and H. Jaffrès, Phys. Rev. B 64, 184420 (2001).

${ }^{4}$ X. Jiang, R. M. Shelby, R. M. Macfarlane, S. R. Bank, J. S. Harris, and S. S. P. Parkin, Phys. Rev. Lett. 94, 056601 (2005).

${ }^{5}$ T. Manago, A. Sinsarp, and H. Akinaga, J. Appl. Phys. 102, 083914 (2007)

${ }^{6}$ Y. Lu, V. G. Truong, P. Renucci, M. Tran, H. Jaffrès, C. Deranlot, J.-M. George, A. Lemaître, Y. Zheng, D. Demaille, P.-H. Binh, T. Amand, and X. Marie, Appl. Phys. Lett. 63, 054416 (2008).

${ }^{7}$ W. H. Butler, X.-G. Zhang, T. C. Schulthess, and J. M. MacLaren, Phys. Rev. B 63, 054416 (2001).

${ }^{8}$ A. G. Aronov and G. E. Pikus, Sov. Phys. Semicond. 10, 698 (1976).

${ }^{9}$ R. Fiederling, M. Keim, G. Reuscher, W. Ossau, G. Schmidt, A. Waag, and L. W. Molenkamp, Nature (London) 402, 787 (1999).

${ }^{10}$ Y. Ohno, D. K. Young, B. Beschoten, F. Matsukura, H. Ohno, and D. D. Awschalom, Nature (London) 402, 790 (1999).

${ }^{11}$ F. Meier and B. P. Zakharchenya, Optical Orientation (North-Holland, Amsterdam, 1984).

${ }^{12}$ Y. Lu, C. Deranlot, A. Vaurès, F. Petroff, J.-M. George, Y. Zheng, and D. Demaille, Appl. Phys. Lett. 91, 222504 (2007).

${ }^{13}$ J. S. Lee and C. Nguyen, Electron. Lett. 37, 504 (2001).

${ }^{14}$ The parasitic effects due to Magnetic Circular Dischroism and to the electron Zeeman splitting in the QW have been measured by photoluminescence spectroscopy under linearly polarized excitation light. We found that these effects yield a circular polarization degree $P_{C}<2 \%$ for $B=0.8 \mathrm{~T}$. This point was also confirmed by a control measurement on a device with a Pt layer instead of $\mathrm{CoFeBo}$

${ }^{15}$ B. L. Liu, P. Renucci, H. Carrère, M. Sénès, X. Marie, T. Amand, J. F. Bobo, C. Fontaine, A. Arnoult, and P. H. Binh, Phys. Status Solidi C 1, 475 (2004).

${ }^{16}$ G. Salis, R. Wang, X. Jiang, R. M. Shelby, S. S. P. Parkin, S. R. Bank, and J. S. Harris, Appl. Phys. Lett. 87, 262503 (2005).

${ }^{17}$ C. Adelmann, X. Lou, J. Strand, C. J. Palmstrom, and P.A. Crowell, Phys. Rev. B 71, 121301(R) (2005).

${ }^{18}$ C. Adelmann, J. L. Hilton, B. D. Schultz, S. McKernan, C. J. Palmstrom, X. Lou, H. S. Chiang, and P. A. Crowell, Appl. Phys. Lett. 89, 112511 (2006).

${ }^{19}$ X. Y. Dong, C. Adelmann, J. Q. Xie, C. J. Palmstrom, X. Lou, J. Strand, P. A. Crowell, J. P. Barnes, and A. K. Petford-Long, Appl. Phys. Lett. 86, 102107 (2005). 